\title{
Enterovirus A71 Genogroups $C$ and $E$ in Children with Acute Flaccid Paralysis, West Africa
}

\section{Maria D. Fernandez-Garcia, Ousmane Kebe, Aichatou D. Fall, Hamet Dia, Ousmane M. Diop, Francis Delpeyroux, Kader Ndiaye}

Author affiliations: Institut Pasteur, Dakar, Senegal

(M.D. Fernandez-Garcia, O. Kebe, A.D. Fall, H. Dia, K. Ndiaye); World Health Organization, Geneva, Switzerland (O.M. Diop); Institut Pasteur, Paris, France (F. Delpeyroux); Institut National de Santé et de La Recherche Médicale, Paris (F. Delpeyroux)

DOI: http://dx.doi.org/10.3201/eid2204.151588

To the Editor: Human enterovirus 71 (EV-A71) of the species enterovirus A, genus Enterovirus, and family Picornaviridae is a serious public health threat because it can cause large outbreaks of hand, foot and mouth disease (HFMD). In addition, EV-A71 has been associated with severe and sometimes fatal neurologic complications that affect mostly infants and children and that range from aseptic meningitis and encephalitis to poliomyelitis-like acute flaccid paralysis (AFP) (1). EV-A71 has been classified into 7 genogroups, A-G, on the basis of the diversity of the nucleotide sequences of the viral protein 1 (VP1) capsid (1,2). Since 1997, increasing epidemic activity of genogroups $\mathrm{B}$ and $\mathrm{C}$ has been reported in the Asia-Pacific region and has caused large HFMD outbreaks with high rates of illness and death. Subgenogroup C2 was identified as the main causal agent associated with cases of fatal encephalitis in the devastating HFMD outbreaks of Taiwan in 1998 and Australia in 1999 (1). Since those outbreaks, $\mathrm{C} 2$ has been frequently reported in countries in Asia, Europe, North America, and South America and has been associated with neurologic complications and fatal infection (1). Although genogroups $\mathrm{E}$ and $\mathrm{F}$ were recently discovered in Africa, the epidemiology of EV-A71 has been largely unexplored in this continent. Only 4 AFP cases associated with EV-A71 infection have been reported in Africa (2-5), and the only outbreak caused by EV-A71 occurred in Kenya in 2000 among a small number of HIV-infected orphans and was attributed to genogroup $\mathrm{C}(6)$.

To investigate the circulation and genetic diversity of EV-A71 in West Africa, we retrospectively analyzed 236 nonpolio enterovirus (NPEV) isolates obtained through routine poliomyelitis surveillance activities at the World Health Organization's Regional Polio Laboratory in Senegal during 2013-2014. Following WHO guidelines (7), the laboratory received and processed stool specimens from 1,600 children with AFP from various West Africa countries. NPEV was found in isolates from most countries except Cape Verde (0/5 specimens): Gambia (8/64), Guinea-Bissau (8/49), Guinea (42/355), Mauritania (20/108), Niger (95/596), and Senegal (63/423). NPEV was initially characterized by amplification of the VP1 capsid protein coding region by using reverse transcription PCR and partial sequencing, as described (8). After molecular typing of all NPEV isolates, we identified 4 new EV-A71 isolates from 4 patients, 1 each from Guinea, Mauritania, Niger, and Senegal (online Technical Appendix Table 1, http://wwwnc.cdc.gov/EID/article/22/4/151588-Techapp1.pdf). BLAST analysis (http://www.ncbi. nlm.nih.gov/) of the partial VP1 nucleotide sequences showed that the isolates from this study shared $96 \%-97 \%$ nt sequence identity with those of other EV-A71 isolates deposited in GenBank.

The complete VP1 nucleotide sequences of the 4 EVA71 isolates were determined by using reverse transcription PCR (GenBank accession nos. KT818793-KT818796; online Technical Appendix Table 2). Sequences were aligned by using ClustalW (http://www.clustal.org). Phylogenetic investigation indicated that the EV-A71 strains detected in 3 of the 4 isolates (from Senegal, Guinea, and Mauritania) clustered within subgenogroup C2. The most closely related EV-A71 strains are not those previously reported from other Africa countries (Madagascar, Cameroon, Nigeria, and Central African Republic) but are strains found in isolates from France, Finland, United Kingdom, Spain, Germany and Netherlands, all isolated during 2006-2010 (Figure). The $\mathrm{C} 2$ clusters from Africa and Europe showed an average of $97.1 \%$ nt identity and $99.8 \%$ aa similarity. Within the Africa $\mathrm{C} 2$ cluster, the nucleotide and amino acid alignments displayed a substantial proportion of conserved positions: 862/891 (96.7\%) and 297/297 (100\%), respectively. Because most enteroviruses evolve at the rate of $\approx 1 \%$ nt substitutions per year in the VP1 region, the sequence divergence of $\approx 3 \%$ suggests that these enteroviruses had been circulating in the region for $\approx 3$ years before they were detected. However, we cannot exclude the possibility that these isolates originated from multiple importation events from abroad, especially from countries in Europe. The fourth isolate (from Niger) clustered within genogroup E, which, before this study, included only 2 complete VP1 sequences from isolates from Central African Republic and Cameroon and an additional partial VP1 sequence from an isolate from Nigeria.

Comparison of complete VP1 amino acid sequences of all EV-A71 strains considered for the phylogenetic analysis showed that $107(36 \%)$ of 297 aa sites were variable. None of the residues found in the variable sites of the Africa strains in our study corresponded to residues previously associated with genogroup $\mathrm{C}$ neurovirulent phenotypes (A170V, N31D, L97R, G145E and D164E) $(9,10)$. The Niger $\mathrm{E}$ isolate showed specific residues (L24M, A170T) that differed from those of other genogroup E isolates. 


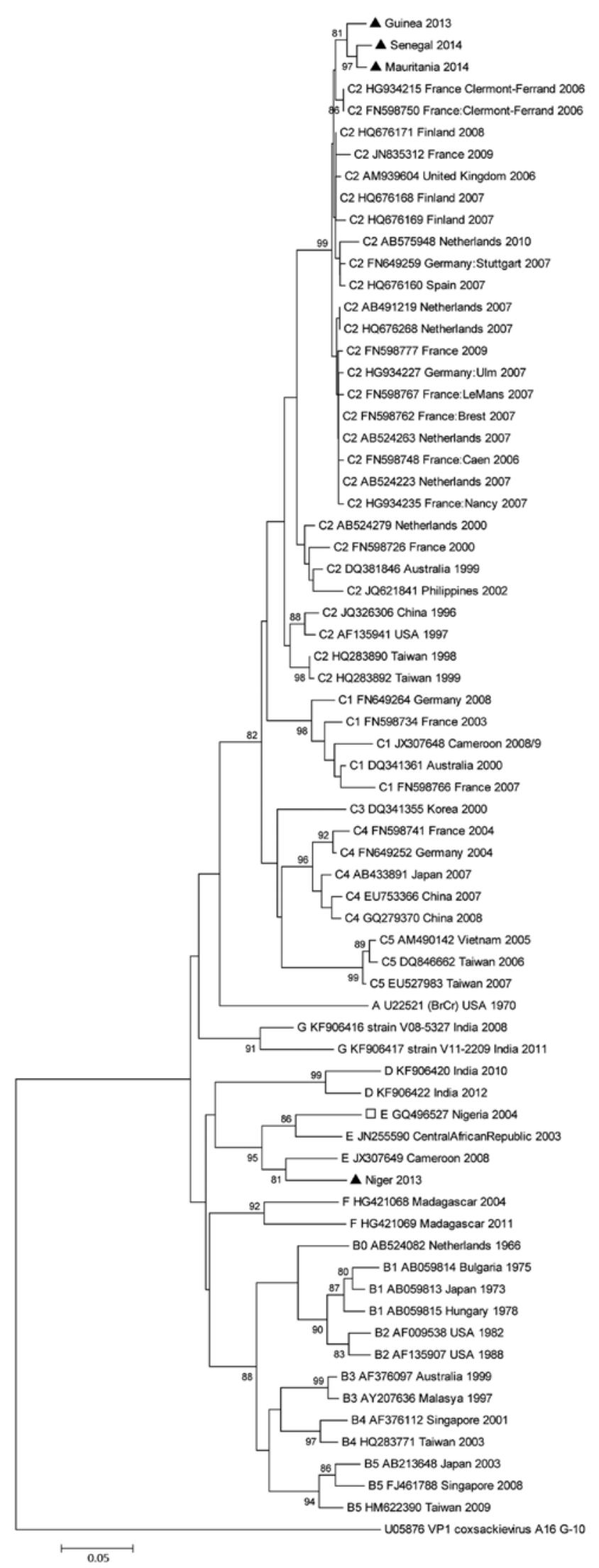

Figure. Phylogenetic tree created with the complete VP1 nucleotide sequences (891 bp in length) of enterovirus A71 isolated from 4 patients with acute flaccid paralysis in West Africa, the most similar nucleotide sequences identified by a search in GenBank by using BLAST (http://www.ncbi.nlm.nih.gov/), and a representative global set of enterovirus A71 sequences belonging to different genogroups and subgenogroups. The coxsackievirus $\mathrm{A} 16$ prototype $\mathrm{G}-10$ sequence was introduced as the outgroup. The tree was inferred with a neighborjoining method that used MEGA5 software (http://www.megasoftware.net/). Distances were computed by using the Kimura 2parameter model. The robustness of the nodes was tested by using 1,000 bootstrap replications. Bootstrap support values $>80$ are shown in nodes. Black triangles indicate the 4 strains from this study. Open square indicates a partial sequence. Scale bar represents nucleotide substitutions per site. Abbreviations of virus names indicate genogroups or subgenogroups/GenBank accession number/origin/year of isolation, respectively. A color version of this figure is available online (http://wwwnc.cdc.gov/EID/ article/22/4/15-1588-F1.htm). 
Our findings highlight the presence of EV-A71 with a high degree of genetic diversity in patients with AFP in West Africa. Future studies about the burden of disease, epidemiologic features, and evolution of EV-A71 in this region of Africa are needed to implement appropriate public health measures.

\section{Acknowledgments}

We are grateful to Romain Volle and Florence Colbère-Garapin for critical review of the manuscript and to Hiroyuki Shimizu for fruitful discussions.

This work was supported by the Pasteur Institute's Transverse Research Programs PTR-276 and PTR-484.

\section{References}

1. Solomon T, Lewthwaite P, Perera D, Cardosa MJ, McMinn P, Ooi MH. Virology, epidemiology, pathogenesis, and control of enterovirus 71. Lancet Infect Dis. 2010;10:778-90. http://dx.doi.org/10.1016/S1473-3099(10)70194-8

2. Bessaud M, Razafindratsimandresy R, Nougairede A, Joffret ML, Deshpande JM, Dubot-Peres A, et al. Molecular comparison and evolutionary analyses of VP1 nucleotide sequences of new African human enterovirus 71 isolates reveal a wide genetic diversity. PLoS One. 2014;9:e90624. http://dx.doi.org/10.1371/ journal.pone.0090624

3. Bessaud M, Pillet S, Ibrahim W, Joffret ML, Pozzetto B, Delpeyroux F, et al. Molecular characterization of human enteroviruses in the Central African Republic: uncovering wide diversity and identification of a new human enterovirus A71 genogroup. J Clin Microbiol. 2012;50:1650-8. http://dx.doi.org/10.1128/JCM.06657-11

4. Oyero OG, Adu FD. Non-polio enteroviruses serotypes circulating in Nigeria. Afr J Med Sci. 2010;39(Suppl):201-8.

5. Sadeuh-Mba SA, Bessaud M, Massenet D, Joffret ML, Endegue MC, Njouom R, et al. High frequency and diversity of species C enteroviruses in Cameroon and neighboring countries. J Clin Microbiol. 2013;51:759-70. http://dx.doi.org/10.1128/JCM.02119-12

6. Chakraborty R, Iturriza-Gomara M, Musoke R, Palakudy T, D'Agostino A, Gray J. An epidemic of enterovirus 71 infection among HIV-1-infected orphans in Nairobi. AIDS. 2004;18:196870. http://dx.doi.org/10.1097/00002030-200409240-00018

7. World Health Organization. Polio laboratory manual. 4th ed. 2004 Aug 31 [cited 2015 Jan 30]. http://apps.who.int/iris/bitstream/10665/68762/1/WHO_IVB_04.10.pdf

8. Bessaud M, Jegouic S, Joffret ML, Barge C, Balanant J, Gouandjika-Vasilache I, et al. Characterization of the genome of human enteroviruses: design of generic primers for amplification and sequencing of different regions of the viral genome. J Virol Methods. 2008;149:277-84. http://dx.doi.org/10.1016/j.jviromet.2008.01.027

9. Zhang B, Wu X, Huang K, Li L, Zheng L, Wan C, et al. The variations of VP1 protein might be associated with nervous system symptoms caused by enterovirus 71 infection. BMC Infect Dis. 2014;14:243. http://dx.doi.org/10.1186/1471-2334-14-243

10. Kataoka C, Suzuki T, Kotani O, Iwata-Yoshikawa N, Nagata N, Ami Y, et al. The role of VP1 amino acid residue 145 of enterovirus 71 in viral fitness and pathogenesis in a cynomolgus monkey model. PLoS Pathog. 2015;11:e1005033. http://dx.doi.org/10.1371/ journal.ppat. 1005033

Address for correspondence: Maria D. Fernandez-Garcia, Pôle de Virologie, Institut Pasteur, 36 Avenue Pasteur, BP220, Dakar, Senegal; email: mdfernandez@pasteur.sn

\section{Hepatitis E Virus Prevalence among Blood Donors, Ouagadougou, Burkina Faso}

\author{
Kuan A. Traoré, Jean Bienvenue Ouoba, \\ Hortense Rouamba, Yacouba K. Nébié, \\ Honorine Dahourou, Frédéric Rossetto, \\ Alfred S. Traoré, Nicolas Barro, Pierre Roques
}

Author affiliations: Centre de Recherche en Sciences Biologique

Alimentair Nutritionnelles, Université Ouaga 1-Pr. Joseph

Ki-Zerbo, Ouagadougou, Burkina Faso (K.A. Traoré, J.B. Ouoba,

A.S. Traoré, N. Barro); Commissariat à l'Energie Atomique, Service d'Immuno-Virologie, Fontenay-aux-Roses, France

(K.A. Traoré, F. Rossetto, P. Roques); INSERM, U1184, Fontenay-aux-Roses (K.A. Traoré, F. Rossetto, P. Roques); Université Paris-Sud XI, Orsay, France (K.A. Traoré, F. Rossetto, P. Roques); Centre Médical de Samandin, Ouagadougou (H. Rouamba); Centre National de Transfusion Sanguine, Ouagadougou (Y.K. Nébié, H. Dahourou)

\section{DOI: http://dx.doi.org/10.3201/eid2204.151728}

To the Editor: The safety of blood product use is continually improving, but blood transfusion remains a challenge in Africa, given the high prevalence of bloodborne pathogens (1). In Africa, the main serologic tests done to reduce blood transfusion risks are for HIV and hepatitis B and $\mathrm{C}$ viruses. However, unknown or emerging pathogens among the population of blood donors, such as hepatitis $\mathrm{E}$ virus (HEV), may also jeopardize transfusion safety.

HEV is emerging as a potential threat to blood safety. High rates of HEV IgG prevalence among blood donors have been found in studies in the United States (7.7\%), England (13.5\%), France (16.6\%), and Spain (19.6\%) (2,3). A study in Iran showed a prevalence of $14.3 \%$ (4), and a study in China showed rates of up to $22.7 \%$ (5). Cases of HEV transmission by transfusion or transplantation have been reported, and recent studies in France and England showed risk for HEV in donated blood ranging from $1 / 2,218$ to $1 / 2,848$ donations $(5$, o).

In Burkina Faso, the prevalence of HEV IgG has been reported as $11.6 \%$ among pregnant women during 2012. Prevalence is $>70 \%$ among butchers, who form a population exposed to pigs, which are a reservoir for $\operatorname{HEV}(7,8)$. To determine whether HEV continues to circulate among human populations outside known at-risk populations, we investigated prevalence of HEV IgG and IgM in the blood donor population of Ouagadougou.

During June and July 2014, we recruited 1,497 first-time blood donors (398 women, 1,099 men) within the National Blood Transfusion Centre in Ouagadougou. Persons 17-65 years of age who weighed $>50 \mathrm{~kg}$ were included (Figure, panel A). Candidate donors were excluded if they had previously received blood transfusions, had jaundice or clinical 